\title{
Das Kardinalat von der Mitte des 12. Jahrhunderts bis zur Mitte des 13. Jahrhunderts (mit besonderer Blickrichtung auf die Iberische Halbinsel)
}

\author{
WERNER MALECZEK
}

Das römische Kardinalskollegium des Hochmittelalters stellt wohl jene herrschaftsorientierte Personengruppe dar, die wie keine andere in ihrer Zusammensetzung, ihrer Organisation und Funktion beschrieben werden kann. Kein anderes geistliches oder weltliches Gremium dieser Zeit, das mit Herrschaftsund/ oder Verwaltungsagenden betraut war, ist prosopographisch besser zu erfassen, kein anderes hinterließ so kräftige Spuren in der Überlieferung. Seit der Mitte des 11. Jahrhunderts wandelte sich bekanntermaßen die Funktion der römischen Kardinäle. Die liturgischen Aufgaben im Dienst der römischen Bischöfe wurden von weit ausgreifenden Verpflichtungen zum Ausbau der päpstlichen Universalmonarchie überlagert, wobei den Inhabern der suburbikarischen Bistümer, den Kardinalbischöfen, zunächst eine prominente Rolle zukam. ${ }^{1}$ Die beiden anderen ordines der Kardinäle, die Kardinalpriester und die Kardinaldiakone, konnten sich in den bewegten Jahrzehnten des Investiturstreites mit seinen Papstschismen, Papstexilaufenthalten und Bedrohungen von außen jenem der Kardinalbischöfe funktionsmäßig angleichen, indem sie sowohl an der Kurie als auch an der Peripherie als Legaten und Verwalter des

1 Die Geschichte des Kardinalskollegiums wird in allen Überblickswerken zur Papstund Kirchengeschichte behandelt, z. B. Machtfülle des Papsttums (1054-1274), hg. von André VAuCHEZ, deutsche Bearbeitung von Odilo ENGELS, Freiburg 1994 (Geschichte des Christentums V); Collin MORRIS: The Papal Monarchy. The Western Church from 1050 to 1250, Oxford 1989; Ian Stuart RoBInSON: The Papacy 10731198. Continuity and Innovation, Cambridge 1990. Nun spezifisch: Geschichte des Kardinalats im Mittelalter, hg. von Jürgen DENDORfER/ Ralf LÜTZELSCHWAB, Stuttgart 2011 (Päpste und Papsttum 39). - Vgl. auch Pierre JugIE: Cardinal (jusqu'au concile de Trente), in: Dictionnaire historique de la papauté, hg. v. Philippe LEVILLAIN, Paris 1994, S. 277-281. - Die rechtliche Seite wird stärker behandelt von Stephan KutTneR: Cardinalis. The history of a canonical concept, in: Traditio 3 (1945) S. 129-214, mit Zusätzen im Neudr. DERS.: The History of Ideas and Doctrines of Canon Law in the Middle Ages, London 1980, Retractationes S. IX 14-18; Carl Gerold FÜRST: Cardinalis. Prolegomena zu einer Rechtsgeschichte des römischen Kardinalskollegiums, München 1967. Weniger Zustimmung fand das unter dem Eindruck des Zweiten Vatikanums geschriebene Buch von Giuseppe AlBERIGO: Cardinalato e collegialità. Studi sull'ecclesiologia tra l'XI e il XIV secolo, Firenze 1969 (Testi e ricerche di scienze religiose 5). 
Patrimoniums zu unentbehrlichen Agenten der zentralisierenden päpstlichen Kirchenherrschaft wurden. ${ }^{2}$ Die Entwicklung des Kardinalskollegiums zum wichtigsten Beraterkreis des Papstes und zum Gremium, das dessen Ansprüche als Oberhaupt der Kirche durchzusetzen half, vollzog sich nicht geradlinig, aber um die Mitte des 12. Jahrhunderts, wo wir unsere Überlegungen einsetzen lassen wollen, lag die Organisationsform und die Funktion weitgehend fest. Das folgende Jahrhundert wird daran noch einiges, aber nicht Fundamentales ändern, die bessere Quellenlage wird einen schärferen Blick erlauben. ${ }^{3}$ Es soll hier sogleich festgehalten werden, daß im folgenden die Kurienkardinäle im Mittelpunkt stehen. Die sogenannten ,auswärtigen Kardinäle', Inhaber von bedeutenden Bischofssitzen oder Abteien, bleiben außer Betracht. Sie sind zahlenmäßig irrelevant und verschwinden um die Mitte des 13. Jahrhunderts. ${ }^{4}$

Nach diesen knappen einleitenden Bemerkungen soll die Beschreibung des Kardinalskollegiums zwischen 1159, dem umstrittenen Beginn des Pontifikats Alexanders III. und Viktors IV., und 1254, dem Ende des Pontifikats Innocenz' IV., in folgenden Punkten erfolgen: Zahlen und soziologischer Querschnitt, rechtliche Stellung, öffentliche Kommunikation, Funktionen und Untergruppen. Abschließend soll den Kardinälen und der Iberischen Halbinsel besonderes Augenmerk gewidmet sein.

2 Vgl. den einleitenden Teil von Rudolf Hüls: Kardinäle, Klerus und Kirchen Roms 1049-1130, Tübingen 1977 (Bibliothek des Deutschen Historischen Instituts in Rom 48).

3 Vgl. Werner MaleczeK: Papst und Kardinalskolleg von 1191 bis 1216 . Die Kardinäle unter Coelestin III. und Innocenz III., Wien 1984 (Publikationen des Historischen Instituts beim Österreichischen Kulturinstut in Rom I/6), bes. S. 207-252 (,Die Entstehung des Kardinalskollegiums in der Zeit des Reformpapsttums. Entwicklungslinien bis zum Ende des 12. Jahrhunderts"); Claudia ZEY: Entstehung und erste Konsolidierung. Das Kardinalskollegium zwischen 1049 und 1143; Werner MALECZEK: Die Kardinäle von 1143 bis 1216. Exkusive Papstwähler und erste Agenten der päpstlichen plenitudo potestatis; Andreas FISCHER: Die Kardinäle von 1216 bis 1304. Zwischen eigenständigem Handeln und päpstlicher Autorität, in: DENDORFER/ LÜTZELSCHWAB: Geschichte (wie Anm. 1), S. 63-225.

4 Vgl. Klaus GANZER: Die Entwicklung des auswärtigen Kardinalats im hohen Mittelalter, Tübingen 1963 (Bibliothek des Deutschen Historischen Instituts in Rom 26). 


\section{Zahlen und soziologischer Querschnitt ${ }^{5}$}

Insgesamt ist eine Gruppe von etwa 170 Männern zu analysieren. Ich stelle zunächst einander gegenüber: die Zahl der Kardinäle bei Pontifikatsbeginn und die Zahl der zu Kardinälen kreierten Männer.

Alexander III. 1159: 30 - 31 neue Kardinäle == Lucius III. 1181: 25 - 15 neue Kardinäle == Urban III. 1185: 26 - keine Kreation == Gregor VIII. 1187: 19 - keine Kreation $==$ Clemens III. 1187: $19-24$ neue Kardinäle $==$ Coelestin III. 1191: 35 - 5 neue Kardinäle == Innocenz III. 1198: $24-30$ neue Kardinäle $==$ Honorius III. 1216: $23-6$ neue Kardinäle $==$ Gregor IX. 1227: 18 - 10 neue Kardinäle == Innocenz IV. 1243: 11 - 15 neue Kardinäle.

Beim Tod dieses Papstes leben noch zwölf Kurienkardinäle. Man konstatiert also ein leichtes Absinken der Zahl, das sich gegen Ende des betrachteten Zeitraumes erheblich beschleunigen wird. Beim Tod Alexanders IV. 1261 fanden sich nur acht Kardinäle in Viterbo zusammen, um einen Nachfolger zu wählen. Diese Tendenz setzt sich in der zweiten Hälfte des 13. Jahrhunderts fort. Erst am Ende des 13. Jahrhunderts konstatiert man durch die massiven Kreationen Coelestins V. und Bonifaz' VIII. eine kräftige zahlenmäßige Steigerung. Dafür läßt sich nur eine hypothetische Begründung finden. Wahrscheinlich konnten die Päpste eine kleinere Personengruppe der mächtigen Mitarbeiter leichter kontrollieren und stützten sich bei der Bewältigung der Regierungsaufgaben mehr und mehr auf untergeordnetes Personal, das in strafferer Ab-

5 Die prosopographischen Zusammenstellungen für diesen Zeitraum sind leider nicht komplett. Hüls: Kardinäle (wie Anm. 2); Barbara ZenKer: Die Mitglieder des Kardinalkollegiums von 1130-1159, Diss. Würzburg 1964; MALECZEK, Papst und Kardinalskolleg (wie Anm. 3); Agostino PARAvicini Bagliani: Cardinali di Curia e ,Familae' cardinalizie dal 1227 al 1254, I-II, Padova 1972 (Italia Sacra 18, 19), sind erheblich breiter gefaßt als die alten Arbeiten von Johannes BRIXIUS: Die Mitglieder des Kardinalkollegiums von 1130 bis 1181, Straßburg 1912 und Elfriede KARTUSCH: Das Kardinalskollegium in der Zeit von 1181 bis 1227, masch. Diss. Wien 1948. Ergänzungen zu Paravicini Bagliani bietet für den Pontifikat Innocenz' IV. Andreas FisCHER: Kardinäle im Konklave. Die lange Sedisvakanz der Jahre 1268 bis 1271, Tübingen 2008 (Bibliothek des Deutschen Historischen Instituts in Rom 118), S. 14-117. Die Lücken im 12. Jahrhundert, d. h. in besonderem Maße der Pontifikat Alexanders III. (1159-1181), werden etwas gefuillt durch Helene TILLMANN: Ricerche sull'origine dei membri del collegio cardinalizio nel secolo XII, in: RSCI 24 (1970) S. 441-464; 26 (1972) S. 313-353; 29 (1975) S. 363-402. Die Lücke im Pontifikat Honorius' III. (1216-1227) wird durch Einzelmonographien ausgeglichen, z. B. Falko NEININGER: Konrad von Urach († 1227). Zähringer, Zisterzienser, Kardinallegat, Paderborn 1994 (Quellen und Forschungen aus dem Gebiet der Geschichte NF 17), oder Nicolas VINCENT: The Letters and Charters of Cardinal Guala Bicchieri, Papal Legate in England, 1216-1218, Woodbridge 1996 (Canterbury and York Society 83). - DeNDORFER/ LÜTZELSCHWAB: Geschichte (wie Anm. 1) haben im Anhang (S. 463-505) eine Liste aller Kardinäle von 1049 bis 1503. 
hängigkeit stand. Obwohl den Kardinälen kein Recht zustand, über die Auswahl ihrer zukünftigen Kollegen mitzubestimmen, ist auch ihr Interesse verständlich, Macht, Einfluß und Einkünfte nicht mit einem großen Personenkreis teilen zu müssen.

Unter soziologischem Querschnitt verstehe ich Feststellungen zu Herkunft, Bildungsstand, Ordenszugehörigkeit, Karrieren bis zur Kardinalskreation. Bei etwa drei Vierteln der Kardinäle sind diesbezügliche Aussagen möglich. Die ganz überwiegende Heimat der Kardinäle ist die Apenninen-Halbinsel, wobei der Schwerpunkt Rom und das heutige Latium in den Jahrzehnten um die Wende zum 13. Jahrhundert uns weiter unten nochmals beschäftigen wird. Aus Ober- und Mittelitalien stammten nachweislich in jedem Pontifikat einige Kardinäle, aus dem Herrschaftsbereich der normannisch-sizilischen Könige sind sie seltener. Von einer Internationalisierung kann nur ansatzweise gesprochen werden. Ein bis drei Franzosen begegnet man unter jedem Papst, die Deutschen, Spanier und Engländer sind hingegen selten.Von den wenigen Iberern wird noch die Rede sein. Eine Ausnahme stellt jedenfalls der Ungar Stephan Vancsa dar, der von Innocenz IV. 1252 ins Kollegium berufen wurde. Im Laufe des 12. Jahrhunderts war immer deutlicher geworden, daß höhere Bildung für eine kuriale Karriere von Vorteil war. Besonders Rechtskenntnisse qualifizierten für die Beteiligung an den stets zahlreicher werdenden Prozessen, aber auch spezifisch theologische Studien konnten förderlich sein, besonders unter den Päpsten, die selbst studiert hatten wie Coelestin III., Innocenz III., wohl auch Gregor IX. und der Jurist Innocenz IV. Die Absolventen von Universitätsstudien nahmen langfristig zu. Beispielsweise führten von den $30 \mathrm{Kar}-$ dinälen, die Innocenz III. ihre Würde verdankten, 14 den Titel eines Magisters oder hatten nachweislich an einer Hohen Schule studiert. Der Anteil der Ordensleute ist - besonders verglichen mit den Zeiten des Reformpapsttums bescheiden. Einige wenige Regularkanoniker stehen einer Handvoll Benediktiner gegenüber; die Zisterzienser sind etwas zahlreicher. Bis um die Mitte des 13. Jahrhunderts schaffte nur ein einziges Mitglied der neuen Bettelorden den Einzug ins Kardinalskollegium. Es ist der berühmte Theologe Hugo von SaintCher, den Innocenz IV. 1244 in seine Umgebung berief. ${ }^{6}$ Der erste Minorit wird erst 1273 kreiert werden, es wird der berühmte Generalminister und große Theologe Bonaventura sein. Was die kirchliche Karriere vor der Kardinalskreation anlangt, so verbieten sich Verallgemeinerungen, außer in einem Bereich: Immer konnten einige Männer niedrigere Dienste an der Kurie zum Karrieresprung nutzen, auffallend viele unter Innocenz III. Fast ein Drittel der von ihm Kreierten waren päpstliche Kapläne gewesen.

6 Vgl. Magdalena BiEnIAK: The soul-body problem at Paris, ca. 1200-1250. Hugh of StCher and his contemporaries, Löwen 2010 (Ancient and Medieval Philosophy I/42); Hugues de Saint-Cher († 1263). Bibliste et théologien, hg. v. Louis-Jacques BATAILLON/ Gilbert DAHAN/ Pierre-Marie Gy, Turnhout 2005 (Bibliothèque d'histoire culturelle du moyen âge 1). 


\section{Rechtliche Stellung}

Es ist eindeutig, daß allein dem Papst die Auswahl der Kardinalskandidaten und die Festsetzung einer Kreation zu den traditionellen Quatemberterminen zusteht. Zeugnisse über die Mitwirkung des Kardinalskollegiums bei der Erhebung neuer Mitglieder sind sehr selten und geben sicher nicht den allgemeinen Usus der von uns betrachteten Epoche wieder. Die Betrauung mit römischen Titelkirchen oder Kardinaldiakonien war eben seit jeher eine Angelegenheit des römischen Bischofs. Die Äußerungen der Kanonisten des 12. Jahrhunderts zu den Kardinälen sind enttäuschend dürftig und entsprechen nicht der Rolle, die die Kardinäle in der täglichen Regierungsarbeit spielten. ${ }^{7} \mathrm{Zu}$ ihrer Entschuldigung muß man aber sagen, daß die wenigen Stellen über Kardinäle im Decretum Gratiani und in den Dekretalen der zweiten Hälfte des 12. Jahrhunderts keine Systematisierung nahelegen. Erst in der zweiten Hälfte des 13. Jahrhunderts wird Hostiensis in seinen Lecturae in quinque libros decretalium eine detailliertere Analyse des Kardinalates liefern, die die Verfassungswirklichkeit besser widerspiegelt. Als er 1262 selbst zur Kardinalswürde aufgestiegen war, betonte er die Befugnisse der Kardinäle zur Mitregierung im Hinblick auf eine kollegiale Kirchenverfassung, welche von einzelnen ekklesiologischen Denkern als Kontrapunkt zur hierokratischen, papalistischen Doktrin immer wieder vertreten wurde. ${ }^{8}$ Definiert war eigentlich nur das Recht der Kardinäle zur Wahl des Papstes durch das Papstwahldekret von 1059 und die Konstitution Licet de vitanda vom III. Lateranum von 1179, so daß sich die Kanonisten hauptsächlich den Kopf über die Rolle der Kardinäle als Wähler und ihre Befugnisse während der Vakanz zerbrachen.

7 Vgl. Alberigo: Cardinalato e collegialità (wie Anm. 1). Die Belege sind gut aufgearbeitet bei Brian TIERnEY: Foundations of the Conciliar Theory. The Contributions of the Medieval Canonists from Gratian to the Great Schism, Cambridge 1955 (Cambridge studies in medieval life and thought NS 4), bes. S. 68ff.; Leiden ${ }^{2} 1998$ (Studies in the History of Christian Thought 81), unveränderter Text, mit einer Einleitung; mit Ergänzungen bei DEMS.: Pope and Council. Some New Decretist Texts, in: Mediaeval Studies 19 (1957) S. 197-218, wiederabgedr. in: DERS.: Church, Law and Constitutional Thought in the Middle Ages, London 1979 (Variorum Reprints CS 90), no. II. Vgl. auch MALECZeK: Papst und Kardinalskolleg (wie Anm. 3), S. 270282.

8 Vgl. James A. WATt: The Constitutional Law of the College of Cardinals: Hostiensis to Johannes Andreae, in: Mediaeval Studies 33 (1971) S. 127-157; DERS.: Hostiensis on Per Venerabilem. The Role of the College of Cardinals, in: Authority and Power. Studies presented to Walter Ullmann, hg. v. Peter LineHAN/ Brian TiERnEY, Cambridge 1980, S. 99-113; Brian TIERNEY: Hostiensis and Collegiality, in: Proceedings of the Fourth International Congress of Medieval Canon Law, Toronto, 21-25 August 1972, hg. v. Stephan KutTner, Città del Vaticano 1976 (Monumenta Iuris Canonici C/5), S. 401-409, wiederabgedr. in: Brian TIERNEY: Rights, laws and infallibility in medieval thought, Aldershot 1997 (Variorum Reprints CS 578) no. X; Roberto GRISON: Il problema del cardinalato nell'Ostiense, in: AHP 30 (1992) S. 125-157. 
Von den elf Päpsten unseres Zeitraumes äußerte sich nur Innocenz III. zur rechtlichen Position der Kardinäle, und der Grundtenor ist immer derselbe: sie beziehen ihre Gewalt allein vom Papst her. In seinem Denken ist für eine Oligarchie der Kardinäle absolut kein Platz. So wie den Bischöfen und den niederen Rängen der kirchlichen Hierarchie ihre Weihegewalt und ihre Jurisdiktion nur als Ausfluß der päpstlichen plenitudo potestatis zukommt, so beruht die Legitimation des Wirkens der Kardinäle allein auf dem Papst. Wenn er sie als membra corporis sui bezeichnet, ist damit keine Gleichrangigkeit gemeint, sondern eine Unterordnung unter das caput, mit dem Innocenz III. immer seine Funktion in der Kirche beschreibt. ${ }^{9}$ Das paulinische Bild vom menschlichen Körper zur Darstellung der Zuständigkeiten innerhalb der Kirche (nach Eph. 5, 30) - in ekklesiologischen Überlegungen des Mittelalters eine ständig wiederholte Formel - wird hier von Innocenz III. auf den Papst und die Kardinäle angewandt: Sie sind Glieder seines Körpers. Damit griff er auf die Terminologie des römischen Rechts zurück, wo die Senatoren manchmal als Teil des kaiserlichen Körpers aufgefaßt werden (C. 9,8,5). Eine Abwandlung des Themas findet sich in einem Brief an das Kapitel von Ravenna aus dem Jahre 1201, der schnell in Dekretalensammlungen aufgenommen wurde und über die Compilatio III in den Liber Extra kam. ${ }^{10}$ Darin werden die Kardinäle als membra capitis bezeichnet, die dem Papst näher als die membra corporis, d. h. die anderen Glieder der Hierarchie, stehen. Aber dies bedeutet keine prinzipielle Aufwertung, die mit einer besonderen Gewalt verbunden wäre. Diese sollte man auch nicht in der Dekretale Per venerabilem vermuten, wo Innocenz III. von den Kardinälen als den sacerdotes levitici generis spricht, die ihm nach dem levitischen Recht als Helfer zur Seite stehen. ${ }^{11}$ Vereinzelt hatten schon Dekre-

9 Die Register Innocenz' III. 1. Pontifikatsjahr, 1198/ 99, bearb. v. Othmar HageneDER/ Anton HAIDACHER, Graz/Köln 1964 (Publikationen der Abteilung für Historische Studien des Österreichischen Kulturinstituts in Rom II/1,1) S. 515f. Nr. 345. Der Brief wurde nicht in Dekretalensammlungen rezipiert.

10 Ursprünglich im heute verlorenen Register des vierten Jahrganges, nur in Dekretalensammlungen: Bernardus Compostellanus 7.6.3, ed. Heinrich SINGER, Die Dekretalensammlung des Bernardus Compostellanus antiquus, in: SAW.PH 171/2, Wien 1914, S. 41; Compilatio III 1.4.3; Liber Extra 1.5.3, ed. Emil FRIEDBERG, Corpus Iuris Canonici II, Leipzig 1879 (Nachdr. Graz 1955), col. 44.

11 Die Register Innocenz' III., 5. Pontifikatsjahr, 1202/1203, bearb. v. Othmar HAGENEDER (u. a.), Wien 1993 (Publikationen des Historischen Instituts beim Österreichischen Kulturinstut in Rom II/1,5), S. 249-255 Nr. 127 (128), hier S. 254 Zl. 3-5; Liber Extra 4.17.13, ed. FriedBerg, II (wie vorige Anm.) col. 714-716. Zu dieser Dekretale, nach der vor allem die Staatslehre Innocenz' III. diskutiert wurde, vgl. letzthin Othmar Hageneder: Anmerkungen zur Dekretale Per venerabilem Innocenz' III. (X 4.17.13), in: Studien zur Geschichte des Mittelalters. Jürgen Petersohn zum 65. Geburtstag, hg. v. Matthias ThumSER (u. a.), Stuttgart 2000, S. 159-173; Deirdre COURTNEY-BATSON, Per venerabilem: from practical necessity to judicial supremacy, in: Pope Innocent III and his World, hg. v. John C. MoORE, Ashgate 1999, S. 287-303 (jeweils mit umfangreichen bibliographischen Angaben). 
tisten des späten 12. Jahrhunderts das Verhältnis vom Papst zu den Kardinälen mit dieser Metapher beschrieben. Aber damit ist keine Aufwertung ihrer Stellung verbunden. Gregor IX. machte im Jahr 1234 ein beachtliches Zugeständnis an das Kardinalskollegium, als er zwei Konstitutionen erließ, die dessen Mitwirkung bei der Verwaltung des Patrimonium Petri zum Ausdruck brachten. Während in der ersten (Habet utilitas stimulos) die Verwaltung der Kirchenstaatsprovinzen künftig nur noch Kardinälen vorbehalten und dem Kollegium zugleich ein Drittel der Einkünfte aus dem Patrimonium zugestanden wurde, räumte der Papst den Mitgliedern des Kardinalskollegs in seinem zweiten Erlaß (Rex excelsus) ein Beratungs- und Zustimmungsrecht bei allen Besitzstandsveränderungen im Kirchenstaat ein (commune fratrum consilium et assensus). ${ }^{12}$ Aber diese Konstitutionen fanden nicht Eingang in Dekretalensammlungen, und es scheint, daß auf sie nur sehr sporadisch zurückgegriffen wurde. ${ }^{13}$ Es ist bemerkenswert, daß Papst Innocenz IV. in seinem etwa 1245 fertiggestellten Apparatus zum Liber Extra Gregors IX. die beiden für die Kardinäle ergiebigen Dekretalen Innocenz' III. so kommentiert, als ob es das Problem ihrer Machtbeteiligung oder des Ursprungs ihrer Kompetenz als Papstwähler oder als engste Mitarbeiter des Papstes gar nicht gäbe. ${ }^{14}$

\section{3. Öffentliche Kommunikation}

Gesten, Zeichen, Gebärden, Rituale, Inszenierung, andere Sektoren nonverbaler Kommunikation bestimmen auch das Auftreten der Kardinäle und signalisieren vor allem den unvergleichlich hohen Rang des Papstes als caput Christianitatis und, davon abgeleitet, die Würde seiner nächsten Mitarbeiter. Dazu einige Beispiele.

Die Unterschriften der Kardinäle auf päpstlichen Privilegien - in der Mitte des 11. Jahrhunderts einsetzend, seit dem Pontifikat Innocenz' II. regularisiert und in drei Kolumnen angeordnet, ab der zweiten Hälfte des 13. Jahr-

12 Les registres de Grégoire IX, ed. Lucien Auvray, I, Paris 1890, S. 945 Nr. 1715, schon vorher bei Augustin THEINER (Ed.), Codex diplomaticus dominii temporalis S. Sedis I, Rom 1861, S. 102f. Nr. 124: de patrimonialibus sine communi fratrum consilio et assensu alienatio nulla fiat, sitque uni facultas, quod ex causa legitima obstaculum libere contradictionis opponat.

13 Die Konstitution Habet utilitas stimulo findet sich allein in der Summa dictaminis des Thomas von Capua. Von dort veröffentlicht durch Karl HAMPE: Eine unbekannte Konstitution Gregors IX. zur Verwaltung und Finanzordnung des Kirchenstaates, in: ZKG 45 (1926) S. 190-197.

14 Commentaria Innocentii Quarti Pontificis Maximi Super Libros Quinque Decretalium, Frankfurt 1570 (Nachdr. Frankfurt 1968), ad X 1.5.3 und X 4.17.13, S. 37f., 481f. - Zu Innocenz IV. als Kanonisten vgl. Kenneth PENNINGTON in der Internet-Fassung der Medieval and Early Modern Jurists: A Bio-Bibliographical Listing (http://faculty. cua.edu/pennington/1140a-z.htm\#Innocent\%20IV, 20.2.2012). 
hunderts im Rückgang begriffen - haben ursprünglich wohl mit Konsens und angedeuteter Mitbestimmung zu tun. Dann aber dienen sie ausschließlich der Steigerung der Feierlichkeit der Papsturkunde, mit der in der Regel nur ein bestehendes Recht bestätigt wurde. Sie machen dem Empfänger sinnenfällig deutlich, daß nicht allein der Papst, sondern auch seine nächsten Mitarbeiter und höchsten Würdenträger mit ihrer individuellen Persönlichkeit das übertragene oder bestätigte Recht garantierten. Nicht allein der Papst, sondern auch die Kardinäle wurden damit beim Empfänger - Bischöfen, Kapiteln, geistlichen Gemeinschaften - in gewisser Weise präsent. ${ }^{15}$

Siegel verwendeten Kardinäle zunächst ausschließlich in ihrer Funktion als päpstliche Legaten. Seit dem frühen 12. Jahrhundert bezeugt, seit etwa 1130 vereinzelt erhalten, schwankt das Siegelbild für einige Jahrzehnte zwischen einem Brustbild nach dem Muster älterer Bischofssiegel und einer symbolischen Darstellung der römischen Titelkirche. In der zweiten Hälfte des 12. Jahrhunderts lassen sich die Kardinäle verstärkt mit den ihnen zustehenden Zeichen ihrer Würde darstellen. Öfters ist dies die Mitra - auch wenn sie nicht Kardinalbischöfe sind -, vereinzelt auch der Bischofsstab, selbst bei Kardinalpriestern und Kardinaldiakonen, womit die hierarchische Gleichstellung, ja

15 Vgl. Ludwig Schmitz-Kallenberg: Die Lehre von den Papsturkunden, in: Grundriß der Geschichtswissenschaft, hg. v. Aloys MeIster, Leipzig/ Berlin 1906, S. 205-215; Harry BRESSLAU: Handbuch der Urkundenlehre für Deutschland und Italien, I, 2. Aufl., Berlin 1912, S. 76-81; Bruno KatTerbach/ Wilhelm M. Peitz: Die Unterschriften der Päpste und Kardinäle in den Bullae maiores vom 11. bis 14. Jahrhundert, in: Miscellanea Franz Ehrle, IV, Rom 1924 (Studi e Testi 40), S. 177-274; Rudolf HIESTAND: Feierliche Privilegien mit divergierenden Kardinalslisten? Zur Diplomatik der Papsturkunden des 12. Jahrhunderts, in: ADipl 33 (1987) S. 239-268; Matthias KORDES: Der Einfluß der Buchseite auf die Gestaltung der hochmittelalterlichen Papsturkunde. Studien zur graphischen Konzeption hoheitlicher Schriftträger im Mittelalter, Hamburg 1993, S. 200-213; Paulius RabiKaUSKAS: Diplomatica pontificia. Praelectionum lineamenta. Editio quinta emendata et aucta, Rom 1994, S. 40-45; Thomas FrENZ: Papsturkunden des Mittelalters und der Neuzeit, 2. aktualis. Aufl., Stuttgart 2000 (Historische Grundwissenschaften in Einzeldarstellungen 2), S. 19-23; Stefan HIRSCHMANN: Die päpstliche Kanzlei und ihre Urkundenproduktion (1141-1159), Frankfurt/ M. 2001 (Europäische Hochschulschriften III/913) S. 39-59; Otfried KRAFFT: Der monogrammatische Schlußgruß (Bene Valete). Über methodische Probleme, historisch-diplomatische Erkenntnis zu gewinnen, in: Papsturkunden des frühen und hohen Mittelalters. Äußere Merkmale - Konservierung - Restaurierung, hg. v. Irmgard FeEs/ Andreas Hedwig/ Francesco Roberg, Leipzig 2011, S. 209-247; Joachim DAHLHAUS: Rota oder Unterschrift. Zur Unterfertigung päpstlicher Urkunden durch ihre Aussteller in der zweiten Hälfte des 11. Jahrhunderts, in: ebd., S. 249-303; Werner MALECZEK: Die eigenhändigen Unterschriften der Kardinäle - ein Spiegelbild ihrer Persönlichkeit? Mit einem Überblick über eigenhändige Unterschriften auf Urkunden vom Frühmittelalter bis ins 13. Jahrhundert, in: Päpstliche Herrschaft im Mittelalter. Funktionsweisen - Strategien - Darstellungsformen, hg. v. Stefan WeINFURTER, Ostfildern 2012 (Mittelalter-Forschungen 38), S. 239-299. 
Überlegenheit über die lokalen Prälaten dokumentiert werden sollte. Seit Alexander III. verfügten wohl alle Kardinäle ohne Ausnahme über ein Siegel. ${ }^{16}$

Vom Zere mo ni e1l, das den Rang der Kardinäle - besonders auf Legationen - unterstrich und als Mittel öffentlicher Kommunikation diente, wissen wir aus dem 12. und 13. Jahrhundert leider wenig. Im Spätmittelalter wird sich dies radikal verändern, und das feierliche, durch ein ausgefeiltes Ritual bestimmte Auftreten der Legaten läßt sich in vielen Details beschreiben. Die Rückprojektion in die von uns betrachtete Zeit erscheint nicht abwegig. Das Auftreten der Legaten muß jedenfalls aufwendig und prunkvoll gewesen sein, denn Kanon 4 des III. Lateranums beschränkte das Gefolge eines Kardinals auf nicht mehr als 20 bis 25 Pferde, und Kanon 33 des IV. Lateranums schärfte dies ein. ${ }^{17}$ Diese beiden Konzilsdekrete betrafen die Prokurationen, das System der Finanzierung von Legationsreisen, das sich seit dem 11. Jahrhundert entwickelt hatte und das einen Rückschluß auf den Aufwand der Legaten erlaubt. Die Prokurationen, die ursprünglich bei Gerichts- und Visitationsreisen des Bischofs oder seiner Beauftragten $\mathrm{zu}$ entrichten waren, mußten von allen kirchlichen Institutionen auch an die päpstlichen Legaten gezahlt werden. Sie waren eine ständige Quelle von Streitigkeiten und Klagen, und päpstliche Befreiungen von dieser Belastung erhielten zur Gänze oder zum Teil einzelne Orden wie die Kartäuser und die Zisterzienser, später dann die Minoriten, aber auch einzelne Klöster und andere geistliche Institutionen zugesagt. ${ }^{18}$ Besonders in England ist das Jammern über die Habgier der Legaten ein ständig wiederkehrendes Motiv der Chronistik. In Rom selbst spielten die Kardinäle im päpstlichen Zeremoniell eine herausragende Rolle, besonders bei den feierli-

16 Zum Folgenden vgl. Werner MALECZeK: Die Siegel der Kardinäle. Von den Anfängen bis zum Beginn des 13. Jahrhunderts, in: MIÖG 112 (2004) S. 177-203; DERS.: Kardinalssiegel und andere Abbildungen von Kardinälen während des 13. Jahrhunderts, in: Die Kardinäle des Mittelalters und der Renaissance, hg. v. Jürgen Dendorfer (im Druck), S. 223-237, wodurch Julian GARDNER: Some Cardinal Seals of the Thirteenth Century, in: Journal of the Warburg and Courtauld Institutes 38 (1975) S. 7296, mit kleinen Ergänzungen von DEMS.: Innocent III and His Influence on Roman Art of the Thirteenth Century, in: Innocenzo III. Urbs et Orbis. Atti del Congresso internazionale, Roma 1998, hg. v. Andrea SOMmerlechner, II, Rom 2003 (Nuovi Studi Storici $55=$ Miscellanea della Società Romana di Storia Patria 44) S. 1245-1260, hier S. 1252-1257 mit Abb. 6-12, ersetzt wird.

17 Conciliorum Oecumenicorum Decreta, ed. Giuseppe Alberigo (et alii), Bologna ${ }^{3} 1973$, S. 213, 250.

$18 \mathrm{Zu}$ den Prokurationen vgl. noch immer Karl RuESs: Die rechtliche Stellung der päpstlichen Legaten bis Bonifaz VIII., Paderborn 1912 (Görres-Gesellschaft, Sektion für Rechts- und Sozialwissenschaft 13), S. 195-201; R. NAZ, in: DDC VII (1965) S. 314324; Carlrichard BRÜHL: Zur Geschichte der procuratio canonica vornehmlich im 11. und 12. Jahrhundert, in: Papato, cardinalato ed episcopato. Le istituzioni ecclesiastiche della „Societas christiana“ dei secoli XI-XII. Atti della quinta Settimana internazionale di studio. Mendola, 26-31 agosto 1971, Mailand 1974 (Pubblicazioni dell’Università cattolica del Sacro Cuore. Miscellanea del Centro di studi medievali 7), S. 419-431. 
chen liturgischen Zeremonien während des Kirchenjahres und an den Wendepunkten des Pontifikates. Die liturgischen Bücher des späten 12. und frühen 13. Jahrhunderts, die den Ablauf der Wahl, die Inthronisation, die Inbesitznahme des Lateranpalastes, die Weihe und Krönung des Papstes, die Prozessionen quer durch Rom und die Liturgien im Laufe des Kirchenjahres und besonders während der Karwoche beschreiben, weisen den Kardinälen stets einen prominenten Platz zu. ${ }^{19}$

\section{Funktionen}

Die wichtigste Aufgabe des Kardinalskollegiums war zweifellos die Papstwahl. ${ }^{20}$ Die Erfahrung des Schismas von 1159 hatte zum schon erwähnten ersten Kanon des III. Laterankonzils, Licet de vitanda, geführt, der nicht nur das exklusive Wahlrecht der Kardinäle, sondern auch die Zweidrittelmehrheit für eine gültige Wahl festschrieb. Bekanntermaßen blieb diese Verfahrensordnung mit geringfügigen Varianten bis in die Jetztzeit gültig. In der zweiten Hälfte des 12. Jahrhunderts kam das Verfahren des scrutinium zur Anwendung, d. h. die Stimmabgabe aller Kardinäle und die Feststellung der Zweidrittelmehrheit, in der ersten Hälfte des 13. Jahrhunderts zogen die Kardinäle bei der Wahl Honorius' III. 1216 das Verfahren des compromissum vor, d. h. die Übertragung des Wahlrechtes auf einen kleinen Personenkreis, der seinerseits einen Kandidaten auswählte. Bei Gregor IX. entstand offensichtlich rasch Einstimmigkeit, bei der mühsamen Wahl Coelestins IV. 1243 kam wieder die Zweidrittelmehrheit zustande. Innocenz IV. wurde nach der Auskunft seines Biographen Niccolò Calvi unanimiter et concorditer gewählt, was jenseits aller Topik auch stimmen

19 Vgl. Bernhard SchimmelPfEnNig: Die Bedeutung Roms im päpstlichen Zeremoniell, in: Rom im hohen Mittelalter. Studien zu den Romvorstellungen und zur Rompolitik vom 10. bis zum 12. Jahrhundert. Reinhard Elze zur Vollendung seines siebzigsten Lebensjahres gewidmet, hg. v. Bernhard SCHimmelpFenNig/ Ludwig SchmugGe, Sigmaringen 1992, S. 47-61; DERS.: Päpstliche Liturgie und päpstliches Zeremoniell im 12. Jahrhundert, in: Das Papsttum in der Welt des 12. Jahrhunderts, hg. v. ErnstDieter HeHL (u. a.), Stuttgart 2002 (Mittelalter-Forschungen 6), S. 263-272; S. TwYMAN: Papal adventus at Rome in the twelfth century, in: Historical Research 69 (1996) S. 233-253.

20 Vgl. Bernhard SchimmelPfenNig: Papst- und Bischofswahlen seit dem 12. Jahrhundert, in: Wahlen und Wählen im Mittelalter, hg. v. Reinhard SCHNEIDER/ Harald ZimmermanN, Sigmaringen 1990, S. 173-196, bes. S. 175-188; Peter Herde: Die Entwicklung der Papstwahl im dreizehnten Jahrhundert, in: ÖAKR 32 (1981) S. 1141. Immer noch nützlich ist Olga JOELSON: Die Papstwahlen des 13. Jahrhunderts bis zur Einführung der Conclaveordnung Gregors X., Berlin 1928 (Historische Studien 178). Im breiteren Zusammenhang vgl. Alberto Melloni: Das Konklave. Die Papstwahl in Geschichte und Gegenwart, Freiburg 2002 (ital. 2001). 
könnte. ${ }^{21}$ In dem uns interessierenden Zeitraum wurden nur Kurienkardinäle zum Papst gewählt.

Die Mitwirkung der Kardinäle an den Regierungshandlungen der Päpste an der Kurie ist in vielfältiger Weise bezeugt, durch chronikalische Nachrichten aller Schattierungen, durch Dichtung und Satire, durch Überlegungen der Kanonisten, aber auch durch die Papsturkunden selbst, in denen die Formel de fratrum nostrorum consilio (mit Varianten) eine vorausgehende Beratung mit dem Kollegium signalisiert. Der Usus der Päpste, ihnen wichtig scheinende Materien mit den Kardinälen zu beraten, schlägt sich seit dem frühen 12. Jahrhundert in der genannten Formel nieder, etwa seit der Mitte des 12. Jahrhunderts erreicht er eine Festigkeit, die das folgende Jahrhundert und darüber hinaus andauern sollte. Ohne eine definierte Regel beriet der Papst bestimmte Fälle mit den Kardinälen - der Begriff ,Konsistorium' sollte als anachronistisch vermieden werden und das allgemeinere ,Rat der Kardinäle' trifft das Phänomen besser -, wobei die unbestimmten Termini ,wichtig' und ,Gewicht der Persönlichkeiten“ für die Auswahl maßgeblich waren. Wenn das Papsttum auch schon früh Tendenzen zur absoluten Herrschaft zeigte, die ihre ideelle Begründung in der vom petrinischen Amt abgeleiteten plenitudo potestatis fand, so vermochte es sich doch der allgemeinen Tendenz zur Beschränkung der Machtfülle durch ein Gremium nicht zu entziehen, abgesehen von pragmatischen Notwendigkeiten, die eine weltumspannende Herrschaft bedingte. Dazu kam noch wegen der stetig anschwellenden, an der Kurie verhandelten Streitfälle der aus dem römisch-kanonischen Gerichtsverfahren abgeleitete Usus, den Richter durch assessores, consiliarii, iurisperiti u. ä. beraten zu lassen. ${ }^{22}$ Nicht nur die Gesamtheit der Kardinäle unterstützte den Papst als beratendes Kollegium und als consilium bei Prozessen, sondern auch einzelne Kardinäle wurden herangezogen, um in der Abwicklung der am kurialen Forum anhängigen Prozesse mitzuwirken. ${ }^{23}$ Die Kardinäle als auditores - der Begriff begegnet als solcher erst unter Coelestin III. - lassen sich ab dem zweiten Drittel des 12. Jahrhunderts gut verfolgen. Da nicht zu erkennen ist, warum der Papst einen bestimmten Kardinal als Auditor einsetzte - persönliche Wertschätzung, Rechtskenntnisse, Vertrautheit mit dem Herkunftsgebiet der Prozeßparteien sind naheliegend -, versteht man, warum sich Petenten an der Kurie intensiv um einzelne Kardinäle bemühten. Nicht wenige Quellen berichten von intensi-

21 Nicolaus de Carbio: Vita Innocentii IV, ed. Francesco PAgNotti, in: ASRSP 21 (1898) S. 80, vgl. Alberto MeLlONI: Innocenzo IV. La concezione e l'esperienza della cristianità come regimen unius personae, Genua 1990 (Istituto per le Scienze religiose di Bologna. Testi e ricerche di scienze religiose NS 4), S. 71.

22 MaleCZeK: Kardinalskollegium (wie Anm. 3), S. 297ff.; Sarah Noethlichs: Das päpstliche Konsistorium im Spiegel der Quellen des 11. bis 13. Jahrhunderts, in: ZRGKanAbt 94 (2008) S. 272-287; FISCHER, Kardinäle von 1216 bis 1304 (wie Anm. 3), S. 181.

23 MaLeCZEK: Kardinalskollegium (wie Anm. 3), S. 325ff.; FISCHER: Kardinäle von 1216 bis 1304 (wie Anm. 3), S. 180. 
vem Lobbyismus unter den Kardinälen und von herumschwirrenden Gerüchten, wer unter ihnen gerade einflußreich sei und wer nicht. - Einzelne Kardinäle leiteten entstehende ,Behörden“ an der römischen Kurie, so die Kanzlei (in relativ kurzen Perioden), die Kammer und die Pönitentiarie. Mit großer Regelmäßigkeit, besonders in der ersten Hälfte des 13. Jahrhunderts, wurde einzelnen Kardinälen das Rektorat in einer der Provinzen des Patrimonium Petri anvertraut.

\section{Untergruppen - Parteiungen}

Es war unvermeidlich, daß sich innerhalb einer Gruppe von 20 bis 30 Männern mit hohen Würden und entwickelten Entscheidungsbefugnissen je nach Interessenslagen, Herkunft, Ausbildung, Verwandtschaftsbeziehungen und natürlich nach Sympathie und Antipathie Untergruppen bildeten, deren $\mathrm{Zu}$ sammensetzung durch den Papst mittels Kreationen und Promotionen beeinflußt werden konnte. Die Unsicherheit über die Verteilung der Einflüsse innerhalb des Kardinalskollegiums ließ von außen her Gerüchte über Machtkämpfe, Fraktionen, Parteiungen entstehen. Aber die diesbezüglichen Nachrichten müssen mit großer Zurückhaltung gelesen werden, und Skepsis ist besonders bei über längere Zeit stabilen Untergruppen mit einem politischen oder geistigen Programm angebracht. Ältere Untersuchungen über das Kardinalskollegium besonders des 13. Jahrhunderts haben versucht, Parteiungen unter den Kardinälen nach politischen Gesichtspunkten nachzuweisen und aus beiläufigen Äußerungen, Anreden und Floskeln des diplomatischen Schriftverkehrs eine ,staufische Partei', eine ,französische Partei' oder eine ,englische Partei' unter den Kardinälen zu konstruieren. ${ }^{24}$ Mit aller Behutsamkeit kann man jedoch bestenfalls zu bestimmten historischen Konstellationen Untergruppen ausmachen. Der Ausbruch des Schismas 1159 könnte von zwei Gruppierungen innerhalb des Kardinalskollegiums beeinflußt worden sein, wobei eine eher dem Kaiser verbunden war und sich vom konstanten Zusammengehen von Kaiser und Papst das Beste versprach und die andere eher der kommunalen Welt Ober- und Mittelitaliens zuneigte. ${ }^{25}$ Ein Beispiel aus dem

24 Ferdinand FeHLING: Kaiser Friedrich II. und die römischen Cardinäle in den Jahren 1227-1239, Berlin 1901 (Historische Studien 21); Berthold SütTERLIN: Die Politik Kaiser Friedrichs II. und die römischen Kardinäle in den Jahren 1239 bis 1250, Heidelberg 1929 (Heidelberger Abhandlungen zur mittleren und neueren Geschichte 58).

25 Ausführlichere Darstellungen des Schismas enthalten die beiden Dissertationen von Timothy Reuter: The Papal Schism, the Empire and the West 1159-1169, Oxford 1975 (ungedruckt), und Willibald MADERTHONER: Die zwiespältige Papstwahl des Jahres 1159, Wien 1978 (Dissertationen der Universität Wien 136), weiters sehr umfangreich Johannes LAUDAGE: Alexander III. und Friedrich Barbarossa, Köln 1997 (Forschungen zur Papst- und Kaisergeschichte des Mittelalters 16). Zusammengefaßt von Werner MALECZEK: Das Schisma von 1159 bis 1177. Erfolgsstrategie und Misser- 
frühen 13. Jahrhundert: In der französischen Königskanzlei wurden zwischen 1216 und 1218 Listen mit Kardinalsnamen in das Kanzleiregister eingetragen und mit den Überschriften Speciales regis und Isti sunt cardinales qui diligunt Fredericum regem et dominum regem Francie versehen. ${ }^{26}$ Bei genauer Betrachtung ergeben sich Widersprüche, so daß man über die tatsächlichen Parteiungen im Kardinalskollegium daraus kaum etwas beziehen kann. Bei der lange währenden Vakanz der Jahre 1268 bis 1271 galt in der wissenschaftlichen Literatur lange Zeit der Antagonismus zwischen einer französisch-angevinischen und einer kaiserlich-staufischen Partei als der Grund für die gegenseitige Lähmung, aber erst die rezente, genaue Untersuchung von Andreas Fischer ergab, daß es viel eher die divergierenden Interessen von römischen Familien und deren Streben nach der Papstwürde waren. ${ }^{27}$ Am naheliegendsten ist es, Untergruppierungen im Kardinalskollegium nach derselben geographischen Herkunft und nach Verwandtschaftsverhältnissen zu suchen. Nur ein Beispiel: Das römische Element ist in ,unserem' Jahrhundert sehr stark vertreten. Grundgelegt wurde dies durch den Römer Paolo Scolari, Papst Clemens III., der nach seiner Aussöhnung mit der Kommune im Jahre 1188 das Kardinalskollegium massiv aufstockte, seine römischen Parteigänger bevorzugte und sich so gegen mögliche antipäpstliche Tendenzen in der kommunalen Führungsschicht absicherte. Seine Nachfolger zeigten immer eine ausgeprägte Bevorzugung der Römer.

\section{Die Kardinäle und die Iberische Halbinsel}

Der Blick auf die Kardinäle und die Iberische Halbinsel in der Zeit von etwa 1150 bis etwa 1250, der hier als Spezialfrage angekündigt wurde, kann kurz ausfallen, denn einerseits gab es in diesem Zeitraum nur drei Kardinäle, die von jenseits der Pyrenäen kamen, und andererseits waren es auch nur wenige Kardinallegaten, die die weite Reise von der römischen Kurie nach Spanien oder Portugal machten.

Der erste der drei Spanier ist Pelagius, der aus León stammte, dort zum Kathedralkapitel gehörte und dem die Historiographie seit dem 17. Jahrhundert den Familiennamen Galvan oder Gaitan gibt, ohne ausreichende Belege zu haben. ${ }^{28}$ Er war ein bedeutender Kanonist, und als solcher dürfte er an der

folgsgründe, in: Gegenpäpste. Ein unerwünschtes Phänomen, hg. v. Harald MüLLER/ Brigitte HoTZ, Wien/Köln/Weimar 2012, S. 165-204.

26 Les registres de Philippe Auguste I: Texte, publ. par James W. BALDWIN, Paris 1992 (Recueil des historiens de la France. Documents financiers et administratifs 7), S. $348 \mathrm{f}$.

27 FISCHER: Kardinäle im Konklave (wie Anm. 5).

28 MALECZEK: Kardinalskollegium (wie Anm. 3), S. 166-170, mit Ergänzungen von DEMS.: Zwischen lokaler Verankerung und universalem Horizont. Das Kardinalskollegium unter Innocenz III., in: Innocenzo III. Urbs et Orbis (wie Anm. 16), S. 154-156. Verstreute Hinweise schon bei Peter LinEHAN: The Spanish Church and the Papacy in 
Kurie bekannt geworden sein. Innocenz III. erhob ihn 1206 oder 1207 zum $\mathrm{KD}$ von S. Lucia in Septasolio, 1211 wurde er KP von S. Cecilia und $1213 \mathrm{~KB}$ von Albano. Aus seinen Funktionen hervorzuheben sind die Legationen ins Lateinische Kaiserreich 1213/ 1215 und unter Honorius III., zu dessen führenden Beratern er zählte, die geistlich-weltliche Leitung des Fünften Kreuzzuges, die ihn vier Jahre lang (1218-1222) von der Kurie fernhielt. Unter Gregor IX. führte er eine Abteilung der Schlüsselsoldaten im Krieg gegen Kaiser Friedrich II. seit 1229 und starb quasi als Gefangener in Montecassino im Januar 1230. Pelagius zählte während seiner Anwesenheit an der Kurie zu den am häufigsten eingesetzten Auditoren bei Rechtsverfahren, unter denen den spanisch-portugiesischen Angelegenheiten ein gewisse Bedeutung zukommt, öfters in Zusammenarbeit mit dem zweiten, gleich zu nennenden Kardinal.

Der zweite spanische Kardinal ist Aegidius, Gil Torres, wahrscheinlich aus Zamora stammend, vor seiner Kreation zum KD von SS. Cosma e Damiano durch Honorius III. im Dezember 1216 Archidiakon von Burgos, der - nach den vielfältigen Aufgaben im kurialen Gerichtswesen und nach seiner Herkunft aus Zamora, dem damals führenden Zentrum kanonistischer Gelehrsamkeit auf der Pyrenäenhalbinsel, zu schließen - ebenfalls juristisch gebildet gewesen sein muß. Gil Torres war überwiegend für spanische und vielleicht auch portugiesische Agenden an der Kurie eingesetzt; 1234 bemühten sich die Kanoniker von Tarragona, ihn zu ihrem Erzbischof zu machen, und 1247 taten die Kanoniker von Toledo das gleiche, beide Male vergeblich. Er wurde zur bevorzugten Anlaufstelle spanischer Petenten und Prokuratoren und erwies sich als großzügig bei der Provision von spanischen Günstlingen mit Pfründen in seiner Heimat. Er scheint aber auch ein enger Vertrauter Innocenz' IV. gewesen zu sein, der ihn zumeist in seiner Umgebung wissen wollte. Legationen freilich erhielt er keine übertragen. Er starb nach einem fast vierzigjährigen Kardinalat im Jahr $1254 .{ }^{29}$

Wilhelm, der dritte der aus Spanien kommenden Kardinäle, war von Geburt aus wahrscheinlich Franzose, der Cluniazenser wurde, ab 1221 das Priorat von S. Juan in Burgos leitete und ab 1227 der Abtei von Sahagún vorstand. Wegen Streitigkeiten mit den Bewohnern von Burgos hielt er sich in der ersten Hälfte der Dreißigerjahre jahrelang an der römischen Kurie auf. Ein zweiter, ebenfalls jahrelanger Aufenthalt in Rom begann 1239 auf Empfehlung des kastilischen Königs Ferdinand und sollte der Friedensvermittlung zwischen Gregor IX. und Kaiser Friedrich II. dienen. Dieser Aufenthalt mündete 1244 in die Erhebung zum KP von SS. XII Apostoli. Ständig in der Umgebung Innocenz' IV., fiel er bis zu seinem Tod in Lyon 1250 kaum auf, und Bezüge

the 13th Century, Cambridge 1971 (Cambridge Studies in Medieval Life and Thought III/4) Kap. 12 „Spaniards at the Curia“, S. 276-321.

29 Linehan: Spanish Church (wie vorige Anm.), S. 276ff.; DERS.: Columpna firmissima: D. Gil Torres, the Cardinal of Zamora, in: Cross, crescent and conversion. Studies on medieval Spain and christendom in memory of Richard Fletcher, hg. v. Simon BARTON/ Peter LiNEHAN, Leiden 2008 (The medieval Mediterranean 73), S. 241-262. 
zu Spanien sind kaum auszumachen. ${ }^{30}$ - Ein vierter Kardinal namens Johannes, dessen von einigen zeitgenössischen Historiographen verwendete Bezeichnung de Toleto ebenfalls nach Spanien weisen könnte, kam in Wirklichkeit aus England. Er gehörte dem Zisterzienserorden an, und der Beiname läßt vielleicht auf ein Studium in Toledo schließen, denn abgesehen von medizinischen Kenntnissen verfügte er über theologische und kanonistische Bildung, und Arabisch konnte er auch. Aber die Zeit vor seiner Kreation 1244 liegt im Dunkeln, und sein Kardinalat, zumeist in der Umgebung der Päpste bis zu seinem Tod 1275 zugebracht, hatte mit der iberischen Welt kaum etwas zu tun, vielmehr mit seinem Orden, mit England und mit Frankreich. ${ }^{31}$

Da die von Kardinälen und anderen Kurialen durchgeführten Legationsreisen auf die Pyrenäenhalbinsel für das 12. Jahrhundert von Ingo Fleisch eingehend beschrieben und jene für das 13. Jahrhundert von Peter Linehan mehrfach untersucht wurden, genügt eigentlich die Aufzählung: Außer dem Kardinal Hyacinth, der 1154/ 55 und 1171-74 weite Gebiete jenseits der Pyrenäen bereiste, ist sein Neffe Gregor, KD von S. Angelo, zu nennen, der 1191-94 und 1196/ 97 zwei große Legationsreisen durchfuhrte. ${ }^{32}$ Die Legation des Petrus von Benevent, KD von S. Maria in Aquiro, in das vom Albigenserkreuzzug heimgesuchte Südfrankreich führte diesen auch kurz über die Pyrenäen zur Stützung des Infanten Jakob nach dem Schlachtentod seines Vaters Peter bei Muret im Jahre 1213. Vielleicht hielten sich auch Honorius' III. Kardinallegaten im Albigensergebiet jeweils kurz jenseits der aragonesischen Grenze auf, um die Minderjährigkeit des Infanten in seinem Sinn zu lenken: Bertrand von SS. Giovanni e Paolo (1217-1219) und Konrad von Urach, KB von Porto (1222-24). Aber die wichtigste und in vielen Quellen dokumentierte Legation nach Spanien und auch Portugal ist jene des Johannes von Abbéville, KB von Sabina, die von Februar 1228 bis Januar 1230 dauerte und deren lang anhaltende Wirkung in mehreren Provinzialsynoden - Valladolid, Salamanca, Lérida - bestand, von denen jene von Lérida am besten zu beschreiben ist, da die Synodalstatuten erhalten blieben. Es ging darum, die am IV. Lateranum beschlossenen allgemein-kirchlichen Konstitutionen auch in den spanischen Königreichen zu implantieren. ${ }^{33}$

30 LINEHAN: Spanish Church (wie vorige Anm.), S. 279.

31 Vgl. FISCHER: Kardinäle im Konklave (wie Anm. 5), S. 56-72.

32 Ingo FleisCH: Rom und die Iberische Halbinsel. Das Personal der päpstlichen Legationen und Gesandtschaften im 12. Jahrhundert, in: Römisches Zentrum und kirchliche Peripherie. Das universale Papsttum als Bezugspunkt der Kirchen von den Reformpäpsten bis zu Innozenz III., hg. v. Jochen JOHRENDT/ Harald MÜLLER, Göttingen 2008 (Neue Abh. der Akademie der Wissenschaften zu Göttingen, phil.-hist. Klasse. NF 2. Studien zu Papstgeschichte und Papsturkunden), S. 135-190.

33 LineHAN: Spanish Church (wie Anm. 29), passim und bes. S. 20-34; DERS.: A papal legation and its aftermath: Cardinal John of Abbeville in Spain and Portugal, 12281229, in: A Ennio Cortese, hg. v. Domenico MAfFeI/ Italo BIROCCHI, Rom 2001, II, S. 236-256. 
Ein Zitat möge als Zusammenfassung und Abschluß dienen. Als Jordanus von Giano, ein Minorit der ersten Generation, in der Rückschau seines Lebens nach 1262 eine Chronik des Ordens seit seinen Ursprüngen verfaßte und besonders die Frühzeit der Bewegung des hl. Franziskus treffend kennzeichnete, widmete er der Vorsprache des Poverello bei Honorius III. im Jahre 1219 ein Kapitel. Er läßt Franziskus im Dialog mit dem Papst dabei sagen: ,Da du ein großer Herr bist und mit vielen wichtigen Dingen beschäftigt, können die Armen nicht oft zu dir kommen und mit dir sprechen, sooft sie es brauchen würden. Viele Päpste hast du mir gegeben. Gib mir einen, mit dem ich sprechen kann, wenn ich es nötig habe, damit er an deiner Stelle meine Anliegen und jene des Ordens anhöre und bespreche.' - ,Viele Päpste hast du mir gegeben ${ }^{6}$ - gemeint sind damit die Kardinäle, die in der Meinung der Zeitgenossen papstgleich wirkten, ausgestattet mit Vollmachten, versehen mit höchstem Prestige, unentbehrliche Mitarbeiter in der Zentrale der Kirche, mächtig und doch nicht so unnahbar wie der Papst selbst. Jordanus gibt hier die gängige zeitgenössische Ansicht wieder: Die Zentrale der Kirche besteht in der Verfassungswirklichkeit aus dem Papst und seinen Kardinälen. Und tatsächlich: obwohl das Papsttum des 13. Jahrhunderts den Anspruch auf seine plenitudo potestatis immer höher auftürmte, ist es ohne das Kardinalskollegium in der Realität der Leitung der Kirche nicht denkbar. ${ }^{34}$

\section{Summary}

Due to the relatively good tradition of sources, the Roman College of Cardinals of the High Middle Ages can be better described than a comparable secular elite around a ruler. Its form of organisation and its function were to a large extent fixed around the middle of the 12th century. In the space of time considered in this paper, the number of members of the College amounts to roughly 170 , within the pontificates lessening from about 30 to about 15 men. For about three quarters of the cardinals, it is possible to make a statement about their origin, educational background, affiliation to a religious order and similar aspects. The outcome of canonistic comments of the 12th and 13th century on the matter of cardinals is disappointingly poor, which does not correspond to the role they played in daily governmental work. Actually, only the cardinals' right to elect the pope was defined by the constitution Licet de vitanda of the Third Lateranum in 1179. The cardinals' media of public communication were for example their signatures affixed to papal privileges, fur-

34 Chronica fratris Jordani, ed. Heinrich Boehmer, Paris 1908 (Collection d'études et de documents 6), c. 14, S. 14: Domine, cum sis magnus dominus et magnis sepe prepeditus negotiis, pauperes ad te accessum habere sepe non possunt nec tibi loqui, quociens necesse habent. Multos mihi papas dedisti. Da unum, cui cum necesse habeo, loqui possim, qui vice tua causas meas et ordinis mei audiat et discutiat. Vgl. Luigi CANETTI, in: DBI 55 (2000) S. 240-243. 
thermore their seals, and an increasingly difficult ceremonial, which allowed them an unchallenged priority compared to other hierarchy members, especially at the beginning of a pontificate or at outstanding occasions. Individual cardinals controlled emerging 'agencies' at the Roman curia. Sub-groups and factions within the College of Cardinals are rather to be assumed than to be proved. As for the cardinals' relationship with the Iberian Peninsula, only three of them were native of the other side of the Pyrenees, and only few cardinal legates travelled the long way to Spain or Portugal. 
